\title{
Role of vitamin C in gastric cancer
}

\section{Takalkar U Vidyadhar1* and Advani Suresh $^{2}$}

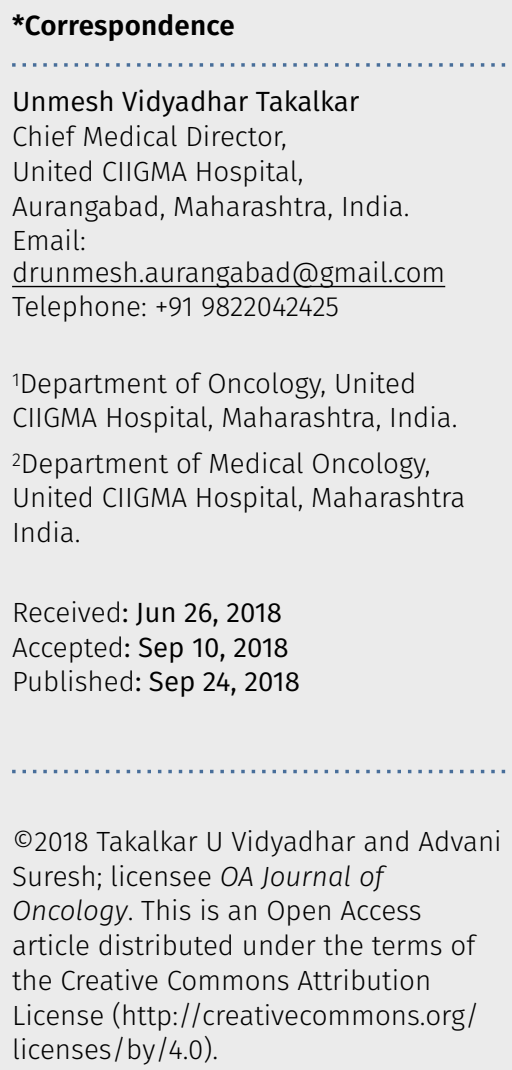

Received: Jun 26, 2018

Accepted: Sep 10, 2018

Published: Sep 24, 2018

C2018 Takalkar U Vidyadhar and Advani Suresh; licensee OA Journal of Oncology. This is an Open Access article distributed under the terms of the Creative Commons Attribution License (http://creativecommons.org/ licenses/by/4.0).

\begin{abstract}
Gastric cancer is a multifactorial disease with complex interplay of environmental and genetic factors. Helicobacter pylori (H. pylori) infestation has been identified as the most important etiological agent in the pathogenesis of gastric cancer. Also, the role of dietary factors that is low consumption of fruits and vegetables have been found to be associated with gastric cancer. Among the dietary factors, antioxidants especially vitamin $\mathrm{C}$ has been found to confer the strongest protection against gastric cancer. Its anti-proliferative and pro-apoptotic action has been suggested in vitro. Because of its antioxidant activity, it protects cells against oxidative DNA damage caused by toxic effects of reactive oxygen species. It also inhibits production of carcinogenic $\mathrm{N}$-nitroso compound in the stomach.

The person with $H$. pylori infection has low levels of vitamin $\mathrm{C}$ in their gastric juice and levels of vitamin $\mathrm{C}$ normalizes on eradication of $\mathrm{H}$. pylori. Vitamin $\mathrm{C}$ levels are high in gastric mucosa and gastric juice, sometimes more than that of in plasma. But gastric pathological conditions cause lowered secretion of vitamin $C$ into gastric juice. Effect of $H$. pylori on vitamin $C$ in gastric juice is reversible and on eradication of $H$. pylori, it returns to normal level. Hence, eradication of $\mathrm{H}$. pylori and chemoprevention with antioxidant supplementation will be an effective preventive strategy to reduce the incidence of gastric cancer and related mortality. Vitamin C and gastric cancer is an area of potential interest for researchers as a preventive measure.
\end{abstract}

Keywords: Vitamin C, H. pylori, gastric cancer.

\section{Introduction}

Gastric cancer is a heterogeneous disease that includes various malignant epithelial lesions with a variety of predisposing conditions and etiological factors. Incidence of gastric cancer varies worldwide between the male and female and across the various countries. It remains the fifth common cancer and third leading cause of cancer related mortality globally. In spite of reduction in the overall incidence of gastric cancer, survival rate is poor around $20 \%$ worldwide because of late presentation at the time of diagnosis [1]. Hence, several researchers have emphasized the importance of effective primary preventive measures for gastric cancer. Identification of the risk factors and chemoprevention of gastric cancer has drawn considerable attraction in the field of clinical oncology research.

Gastric cancer is a multifactorial disease with complex interplay of environmental and genetic factors. Common risk factors are old age, male sex, tobacco smoking, family history, obesity, gastroesophageal reflux disease (GERD). Helicobacter pylori (H. pylori) infestation has been identified as the most important etiological agent in the pathogenesis of gastric cancer. $H$. pylori is 
responsible for distal gastric cancer through different mechanisms like inflammation, genetic instability, epigenetic alterations [2]. Also, the role of dietary factors that is low consumption of fruits and vegetables have been found to be associated with gastric cancer [3]. Among the dietary factors, antioxidants have been found to confer the strongest protection against gastric cancer and by far the most effective of these is vitamin C. Fresh vegetables and fruits contain a wide variety of antioxidants. The causal association between deficiency of vitamin $\mathrm{C}$ and gastric cancer has been documented in literature.

\section{Vitamin C and Gastric Cancer - Possible Link}

Vitamin C or ascorbic acid, a water-soluble vitamin possesses antioxidant activity. It is an acidic molecule with strong reducing property. Because of its antioxidant activity, it protects cells against oxidative DNA damage caused by toxic effects of reactive oxygen species due to $H$. pylori infection [4]. Vitamin C may inhibit the growth of H. pylori and regulate immune response towards $H$. pylori infection. It also inhibits production of carcinogenic $\mathrm{N}$-nitroso compound in the stomach. Its antiproliferative and pro-apoptotic action has been suggested in vitro [5]. The person with $H$. pylori infection has low levels of vitamin $\mathrm{C}$ in their gastric juice and levels of vitamin $C$ normalizes on eradication of $H$. pylori. Vitamin $\mathrm{C}$ levels are high in gastric mucosa and gastric juice, sometimes more than that of in plasma. But gastric pathological conditions cause lowered secretion of vitamin C into gastric juice [6]. Vitamin $C$ cannot be synthesized in human beings hence must be supplied through dietary sources. Citrus fruits, rich in vitamin $\mathrm{C}$ have been found to have protective effect against the risk of development of gastric cancer.

Numerous researchers reported association of higher dietary intakes of vitamin $\mathrm{C}$ with decreased risk of gastric cancer because of its interrelation with $H$. pylori [7]. There is limited epidemiological evidence about the reduction of gastric cancer prevalence on supplementation of high doses of vitamin C. Lam et al investigated the association between prediagnostic serum vitamin $\mathrm{C}$ level and risk of incident gastric adenocarcinoma in high risk Chinese case-cohort study. Their results showed significant inverse association between higher plasma vitamin $\mathrm{C}$ concentration and risk of gastric cancer. They also documented $27 \%$ reduction in risk of gastric cancer among individuals with normal vitamin C concentration [8]. M Jenab and colleagues investigated the association between plasma and dietary vitamin $\mathrm{C}$ levels with the risk of gastric cancer in a case-control study, which was nested within EPIC-EURGAST large cohort involving 10 European countries. They observed inverse gastric cancer risk in the highest versus lowest quartile of plasma vitamin C. Subjects consuming more red and processed meats had more pronounced inverse association which may be due to increased production of endogenous $\mathrm{N}$-nitroso compound [9]. Some of the small sampled case-control studies reported $30-70 \%$ reduction in the prevalence of gastric cancer among people having the high intake of vitamin C. In cancer Prevention Study, participants with higher intake of vitamin $C$ for short duration at the time of enrollment were observed to have reduced risk of gastric cancer [10]. Ma JL et al reported statically significant reduction in gastric cancer mortality in study group who received supplementation of vitamin $C$, vitamin $E$ and selenium, while no effect observed on incidence of gastric cancer [11]. It may be advisable to increase intake of vitamin $C$ enriched food for prevention of gastric cancer. In one of Indian study, researchers observed significantly lower levels of plasma vitamin $\mathrm{C}$ among gastric cancer patients as compared to healthy controls [12].

\section{H. pylori and Vitamin C in Gastric Cancer}

Chronic infection of $\mathrm{H}$. pylori has been identified as a major risk factor for gastric cancer. It can lower the levels of vitamin $\mathrm{C}$ by reducing its systemic bioavailability and concentration of vitamin $C$ in gastric juice. The low dietary intake of vitamin $\mathrm{C}$ rich food can enhance the progression of gastric dysplasia to gastric cancer and supplementation of vitamin $C$ can increase rate of regression of gastric premalignant lesions [6,13]. Zhang Z-W investigated in-vitro effects of vitamin $C$ on gastric cancer cells and $H$. pylori. They found inhibitory effect of ascorbic acid on growth of gastric cell growth and $\mathrm{H}$ pylori induced cell cycles but this inhibitory effect was lost among patients with $\mathrm{H}$. pylori infection [7]. Vitamin $\mathrm{C}$ in gastric juice confers protection against 
gastric cancer but $H$. pylori significantly reduce its concentration. Gastric juice vitamin C levels vary according to methods of estimation and the subjects involved in the studies. The limit has been described in the range of 50-400 uM. Effect of $H$. pylori on vitamin $\mathrm{C}$ in gastric juice is reversible and on eradication of $\mathrm{H}$. pylori, it returns to normal level $[14,15]$ Hence, eradication of $H$. pylori and chemoprevention with antioxidant supplementation will be an effective preventive strategy to reduce the incidence of gastric cancer and related mortality. Among Korean population dietary vitamin C intake was found to have chemopreventive effect that may reduce risk of gastric cancer. But among $H$. pylori infected person, this protective effect is lost [16]. Plummer $M$ and colleagues determined the effect of vitamin C, E and beta-carotene on the prevention for protection against precancerous lesions of gastric cancer. But these antioxidant micronutrients could not be proved as effective tools for chemoprevention of gastric cancer [17]. Vitamin C deficiency is linked to $H$. pylori associated gastritis and high risk of gastric cancer. Some of the observational studies and meta-analyses supported protective effects of vitamin $C$ against gastric cancer. $[2,18]$. However, some researchers reported inconsistent association between vitamin $\mathrm{C}$ and gastric cancer [19-22]. Another important concern in chemoprevention with vitamin $C$ is its dose to be administered. Kim et al found $170 \mathrm{mg}$ per day vitamin $\mathrm{C}$ to be beneficial for protection against gastric cancer among high-risk patients with $\mathrm{H}$. pylori infection [2]. To study the effect of intravenous ascorbic acid on progression free and overall survival and response rate among advanced gastric cancer cases, an interventional clinical trial in phase III as first line therapy is undergoing [23].

\section{Conclusion}

Vitamin $\mathrm{C}$ and gastric cancer is an area of potential interest for researchers as a preventive measure. $\mathrm{H}$. pylori is a major risk factor to promote the development of gastric cancer almost among $65-80 \%$ of the cases. Levels of vitamin C in gastric juice are reduced among people with $H$. pylori infection. Vitamin $C$ has shown the beneficial protective role among high-risk population in prevention of gastric cancer for which $H$. pylori is a significant risk factor. The role of vitamin $\mathrm{C}$ in the chemoprevention of gastric cancer needs to evaluate among high-risk population. It needs wellconducted population based prospective clinical trials.

\section{References}

1. Ferlay J, Soerjomataram I, Dikshit R, et al. (2015) Cancer incidence and mortality worldwide: sources, methods and major patterns in GLOBOCAN 2012. Int J Cancer. 136: E359-E386.

2. Feiz HR, Mobarhan S. (2002) Does vitamin C intake slow the progression of gastric cancer in Helicobacter pylori-infected populations? Nutr Rev. 60: 34-36.

3. Parisa Karimi, Farhad Islami, Sharmila Anandasabapathy, et al. (2014) Freedman and FarinKamangar Gastric Cancer: Descriptive epidemiology, risk factors, screening, and prevention. Cancer Epidemiol Biomarkers Prev. 23: 700-713.

4. Pathak SK, Sharma RA, Steward WP, et al. (2005) Oxidative stress and cyclooxygenase activity in prostate carcinogenesis: targets for chemopreventive strategies. Eur J Cancer. 41: 61-70.

5. Kim HJ, Kim MK, Chang WK, et al. (2005) Effect of nutrient intake and Helicobacter pylori infection on gastric cancer in Korea: a case-control study. Nutr Cancer. 52: 138-146.

6. You WC, Zhang L, Gail MH, et al. (2000) Gastric dysplasia and gastric cancer: Helicobacter pylori, serum vitamin C, and other risk factors. J Natl Cancer Inst. 92: 1607-1612.

7. Zhang Z-W, Abdullahi M, Farthing MJG. (2002) Effect of physiological concentrations of vitamin $C$ on gastric cancer cells and Helicobacter pylori. Gut. 50: 165-169.

8. Lam TK, Freedman ND, Fan J-H, et al. (2013) Prediagnostic plasma vitamin $\mathrm{C}$ and risk of gastric adenocarcinoma and esophageal squamous cell carcinoma in a Chinese population. The American Journal of Clinical Nutrition. 98: 1289-1297.

9. Jenab M, Riboli E, Ferrari P, et al. (2006) Plasma and dietary vitamin $C$ levels and risk of gastric cancer in the European Prospective Investigation into Cancer and Nutrition (EPICEURGAST). Carcinogenesis. 27: 2250-2257.

10. Jacobs EJ, Connell CJ, Collough ML, et al. (2002) Vitamin C, Vitamin E, and Multivitamin Supplement Use and Stomach Cancer Mortality in the Cancer Prevention Study II Cohort. Cancer Epidemiol Biomarkers Prev. 11: 35-41.

11. Ma JL, Zhang L, Brown LM, et al. (2012) Fifteen-Year Effects of Helicobacter pylori, Garlic, and Vitamin Treatments on Gastric Cancer Incidence and Mortality. J Natl Cancer Inst. 104: 488-492.

12. Sahu SK, Giri R. (2017) Study of serum vitamin -C levels in Carcinoma Stomach. IOSR JDMS. 16: 10-12.

13. Correa P, Fontham ET, Bravo JC, et al. (2000) Chemoprevention of gastric dysplasia: randomized trial of antioxidant supplements and anti-helicobacter pylori therapy. J Natl Cancer Inst. 92: 1881-1888.

14. Dabrowska UE, Dzieniszewski J, Jarosz M, et al. (2002) Vitamin C concentration in gastric juice in patients with precancerous lesions of the stomach and gastric cancer. Med Sci Monit. 8: CR96-CR103.

15. Zhang ZW, Farthing MJ. (2005) The roles of vitamin C in Helicobacter pylori associated gastric carcinogenesis. Chin J Dig Dis. 6: 53-58.

16. Kong P, Cai Q, Geng Q, et al. (2014) Vitamin Intake Reduce the Risk of Gastric Cancer: Meta-Analysis and Systematic Review of Randomized and Observational Studies. PLOS ONE. 9: e116060.

17. Plummer M, Vivas J, Lopez G, et al. (2007) Chemoprevention of precancerous gastric lesions with antioxidant vitamin supplementation: a randomized trial in a high-risk population. J Natl Cancer Inst. 99: 137-146. 
18. Hoang BV, Lee J, Choi IJ, et al. (2016) Effect of dietary vitamin C on gastric cancer risk in the Korean population. World J Gastroenterol. 22: 6257-6267.

19. Bjelakovic G, Nikolova D, Simonetti RG, et al. (2004) Antioxidant supplements for prevention of gastrointestinal cancers: a systematic review and meta-analysis. Lancet. 364:1219-1228.

20. López-Carrillo L, López-Cervantes M, Ward MH, et al. (1999) Nutrient intake and gastric cancer in Mexico. Int J Cancer. 83: 601-605.
21. Pelucchi C, Tramacere I, Bertuccio P, et al. (2009) Dietary intake of selected micronutrients and gastric cancer risk: an Italian case-control study. Ann Oncol. 20: 160-165.

22. Epplein M, Shu XO, Xiang YB, et al. (2010) Fruit and vegetable consumption and risk of distal gastric cancer in the Shanghai Women's and Men's Health studies. Am J Epidemiol. 172: 397-406.

23. Phase III Study of IV Ascorbic Acid in Combination With XELOX vs Treatment With XELOX Alone as First-line Therapy for Advanced Gastric Cancer NCT03015675. 